\title{
Risk factors for deep vein thrombosis even using low-molecular-weight heparin after total knee arthroplasty
}

\author{
Joon Kyu Lee ${ }^{1,2^{*}}$, Kee Byoung Lee ${ }^{3}$, Joong $\|$ Kim$^{4}$, Gun Tae Park ${ }^{5}$ and Young Chang Cho
}

\begin{abstract}
Background: With an increase in deep vein thrombosis (DVT) following total knee arthroplasty (TKA) in the Asian population, most surgeons today use a form of prophylactic anticoagulant agents in patients after TKA. Nevertheless, DVT occasionally develops even in these patients with prophylaxis. The purpose of this study was to identify the risk factors for DVT after TKA in cases of postoperative low-molecular-weight heparin (LMWH) use.

Methods: We designed a retrospective study with 103 patients who underwent primary TKA. From the second postoperative day, $60 \mathrm{mg}$ of LMWH was subcutaneously injected into the patients daily. On the seventh postoperative day, patients had computed tomography angiography to check whether they had DVT. Regarding risk factors, we investigated patients' gender, age, surgical site (unilateral/bilateral), body mass index, method of anesthesia, preoperative hypertension, diabetes, hypercholesterolemia status, and prothrombin time/international normalized ratio from electronic medical records. We analyzed the statistical significance of these risk factors.
\end{abstract}

Results: Statistically significant factors in the single-variable analysis were surgical site (unilateral/bilateral), body mass index, preoperative hypertension status, and anesthesia method. Multiple logistic regression analysis with these factors revealed that the surgical site (unilateral/bilateral, $p=0.024)$ and anesthesia method $(p=0.039)$ were significant factors for the occurrence of postoperative DVT after TKA.

Conclusions: Patients undergoing simultaneous bilateral TKAs and patients undergoing TKA with general anesthesia need more attention regarding DVT even with chemoprophylaxis using LMWH after TKA.

Keywords: Deep vein thrombosis, Total knee arthroplasty, Anticoagulant agent, Low-molecular-weight-heparin, Risk factor

\section{Introduction}

Deep vein thrombosis (DVT) is one of the typical complications that can occur after total knee arthroplasty (TKA) $[1,2]$. The management of DVT has long been widely debated. While some insist that calf DVT only needs to be closely monitored, others support some

\footnotetext{
* Correspondence: ndfi@naver.com

'Department of Orthopaedic Surgery, Konkuk University Medical Center, 120-1, Neungdong-ro, Gwangjin-gu, Seoul 05030, South Korea

${ }^{2}$ Research Institute of Medical Science, Konkuk University School of Medicine, 120-1, Neungdong-ro, Gwangjin-gu, Seoul 05030, South Korea

Full list of author information is available at the end of the article
}

form of treatment to prevent proximal transmission, which may even lead to life-threatening pulmonary embolism (PE) [3-7]. Numerous attempts have been made to prevent these complications, and various studies have been conducted on this topic [3, 8-10]. Usually, anticoagulant agents are used with or without other methods to prevent DVT. However, quite a few surgeons have been reluctant to use these agents because of bleeding, swelling, delayed healing of the incision site, and increased risk of infection $[11,12]$.

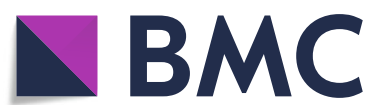

Part of Springer Nature
(9) The Author(s). 2021 Open Access This article is licensed under a Creative Commons Attribution 4.0 International License, which permits use, sharing, adaptation, distribution and reproduction in any medium or format, as long as you give appropriate credit to the original author(s) and the source, provide a link to the Creative Commons licence, and indicate if changes were made. The images or other third party material in this article are included in the article's Creative Commons licence, unless indicated otherwise in a credit line to the material. If material is not included in the article's Creative Commons licence and your intended use is not permitted by statutory regulation or exceeds the permitted use, you will need to obtain permission directly from the copyright holder. To view a copy of this licence, visit http://creativecommons.org/licenses/by/4.0/ The Creative Commons Public Domain Dedication waiver (http://creativecommons.org/publicdomain/zero/1.0/) applies to the data made available in this article, unless otherwise stated in a credit line to the data. 
For Asians, there have been several studies that reported a reduced rate of DVT after TKA compared to the Western population $[4,5,13-18]$. Therefore, there were debates on whether prophylactic treatment for DVT is actually needed after TKA for Asians [6, 19-21]. However, studies have shown that the DVT rate after TKA in the Asian population is increasing, with one study reporting a DVT incidence of up to $62.5 \%$ without prophylaxis [22-24]. Because serious and fatal results can happen if DVT is not treated properly [2], it is now widely accepted that some forms of DVT prevention should be performed, even though some forms of DVT prevention is not up to Western countries [21].

Although these anticoagulant agents certainly reduce DVT occurrence to some extent, there are occasions of DVT occurrence even with the usage of these agents [6]. There are several factors considered to be critical to the occurrence of DVT even with the prophylaxis, but there is a lack of literature reporting factor analysis for DVT in this situation, especially in the Asian population. Chung et al. reported DVT incidence after TKA using postoperative factor $\mathrm{Xa}$ inhibitor in Koreans, but they also acknowledged insufficient data in Asians regarding the DVT incidence with chemoprophylaxis [6].

The purpose of this study was to identify the risk factors for DVT after TKA in cases of postoperative chemoprophylaxis.

\section{Materials and methods Study subjects}

Patients who underwent primary TKA between March 2014 and June 2017 for degenerative osteoarthritis were subjects of this study. Patients taking antiplatelet agents or anticoagulant drugs more potent than aspirin because of their underlying cerebrovascular disease or heart disease were excluded from the study. Patients on low-dose aspirin were guided to stop taking aspirin seven days before the operation. Patients receiving second-stage TKAs were also excluded regardless of previous postoperative DVT status. A total of 103 patients were included in this study.

\section{Surgical information}

A single surgeon carried out all the surgeries on the subjects. An identical TKA procedure was performed in all cases. A midline skin incision, paramedial arthrotomy was performed. Only posterior-stabilized implants with a fixed bearing design were used. All implants were fixed with a cementing technique. Patella resurfacing was not performed. No navigation devices or patient-specific instruments were used. Tranexamic acid was not used at any time prior to, during, or after surgery.

\section{Chemoprophylaxis}

We used low-molecular-weight heparin (LMWH, enoxaparin sodium, Clexane inj. $60 \mathrm{mg} / 0.6 \mathrm{ml}$, Sanofi-Aventis Korea) after TKA for the prevention of DVT along with some mechanical devices [25]. After the operation, we used $60 \mathrm{mg}$ of LMWH daily by injecting it into the subcutaneous layer after removal of the drain, which took place on the second postoperative day. Stockings and pneumatic mechanical pumps were used immediately after the operation.

\section{Rehabilitation}

Range-of-motion (ROM) exercise of the operated knee and walker-assisted ambulation were begun on the second postoperative day. From the fourth postoperative day, routine postoperative rehabilitation treatment, such as gentle passive ROM exercise, parallel-bar gait training, and quadriceps-strengthening exercise, was given daily in the physical therapy room. The rehabilitation protocol was identical for both unilateral and bilateral cases.

\section{Evaluation}

Every patient had lower leg computed tomography (CT) angiography on the seventh postoperative day for the evaluation of DVT. One experienced radiologist confirmed the DVT diagnoses. DVT was defined as a thrombus either in the femoral, superficial femoral, popliteal vein, or veins distal to the popliteal vein. Regarding risk factors for DVT, gender, age (divided into three groups: 64 years and 364 days, 65 years and 0 days to 74 years and 364 days, 75 years and 0 days and older), surgical site (unilateral/bilateral), body mass index (BMI, divided into three groups: under $25 \mathrm{~kg} / \mathrm{m}^{2}, 25-30 \mathrm{~kg} / \mathrm{m}^{2}$, and $30 \mathrm{~kg} / \mathrm{m}^{2}$ or more), type of anesthesia, the preoperative status of hypertension, diabetes, and hypercholesterolemia, and prothrombin time/international normalized ratio (PT INR, divided into four groups: under 0.95, $0.95-1.00,1.00-1.05,1.05$ or more) were checked from the electronic medical records. The factors were selected through a literature review.

\section{Statistical analysis}

Single-variable analyses with the chi-square test or Fisher's exact test were performed to evaluate the relationship of each factor to DVT. Then, with the factors that had significant relationships $(p<0.2)$, multiple logistic regression analyses were done to determine the factors that had a significant impact on the occurrence of DVT after TKA [26-28]. Analyses of unilateral TKA cases were additionally performed. A $p$-value of $<0.05$ was considered significant.

The ethics committee of the Hallym University Sacred Heart Hospital approved this study (2017-I140). Written 
informed consent was exempted by the Institutional Review Board (IRB).

\section{Results}

Among the 103 patients enrolled in this study, 22 patients had positive DVT results. Sixteen patients had DVT only at the veins distal to the popliteal vein. There was one patient each with DVT only at the femoral vein and DVT only at the superficial femoral vein. Two patients had DVT at the popliteal vein alone, and two patients had DVT at both the popliteal vein and the vein distal to the popliteal vein.

In the single-variable analyses, surgical site (unilateral/ bilateral $) \quad(p=0.005)$, BMI $(p=0.043)$, preoperative hypertension $(p=0.078)$, and type of anesthesia ( $p=$ 0.006) were the meaningful factors (Table 1). A multiple logistic regression analysis with these factors revealed that the unilateral/bilateral surgical site $(p=0.024)$ and type of anesthesia $(p=0.039)$ were significant factors for the occurrence of postoperative DVT after TKA (Table 2).
In analyses including only the unilateral cases, gender $(p=0.085)$, BMI $(p=0.021)$, preoperative hypertension $(p=0.156)$, and type of anesthesia $(p=0.084)$ were similarly the meaningful factors in the single-variable analyses (Table 3). However, a multiple logistic regression analysis with these factors revealed no truly significant factor for the occurrence of postoperative DVT (Table $4)$.

\section{Discussion}

This study showed that patients who underwent simultaneous bilateral TKAs and patients with general anesthesia during TKA had a significantly higher risk of DVT after TKA even with LMWH use for chemoprophylaxis of DVT. Therefore, these patients should be carefully monitored for DVT after TKA.

It has long been widely debated whether prophylactic treatment for DVT is needed after TKA in Asians [4, 6, 19-21]. In earlier studies, the results were mostly against the use of prophylactic treatments unless symptomatic DVT developed [19]. However, more recently, studies showed that the DVT rate after TKA in the Korean

Table 1 Single-variable analysis results of factors for the development of deep vein thrombosis

\begin{tabular}{|c|c|c|c|c|}
\hline Factor & & Positive & Negative & $p$ value \\
\hline Deep vein thrombosis & & 22 & 81 & \\
\hline \multirow[t]{2}{*}{ Gender } & Male & 4 & 8 & $0.279^{*}$ \\
\hline & Female & 18 & 73 & \\
\hline \multirow[t]{3}{*}{ Age (years) } & $<65$ & 3 & 21 & $0.773^{* *}$ \\
\hline & $\geq 65$ to $<75$ & 16 & 35 & \\
\hline & $\geq 75$ & 3 & 25 & \\
\hline \multirow[t]{2}{*}{ Unilateral/bilateral } & Unilateral & 15 & 74 & $0.005^{* *_{a}}$ \\
\hline & Bilateral & 7 & 7 & \\
\hline \multirow[t]{3}{*}{ Body mass index $\left(\mathrm{kg} / \mathrm{m}^{2}\right)$} & $<25$ & 5 & 38 & $0.043^{* *_{a}}$ \\
\hline & $\geq 25$ to $<30$ & 13 & 35 & \\
\hline & $\geq 30$ & 4 & 8 & \\
\hline \multirow[t]{2}{*}{ Type of anesthesia } & Epidural & 3 & 37 & $0.006^{* a}$ \\
\hline & General & 19 & 44 & \\
\hline \multirow[t]{2}{*}{ Hypertension } & + & 18 & 48 & $0.078^{* a}$ \\
\hline & - & 4 & 33 & \\
\hline \multirow[t]{2}{*}{ Diabetes } & + & 3 & 20 & $0.389^{*}$ \\
\hline & - & 19 & 61 & \\
\hline \multirow[t]{2}{*}{ Hypercholesterolemia } & + & 4 & 9 & $0.468^{*}$ \\
\hline & - & 18 & 72 & \\
\hline \multirow[t]{4}{*}{ Prothrombin time/international normalized ratio } & $<0.95$ & 8 & 29 & $0.554^{* *}$ \\
\hline & $\geq 0.95$ to $<1.00$ & 8 & 22 & \\
\hline & $\geq 1.00$ to $<1.05$ & 4 & 19 & \\
\hline & $\geq 1.05$ & 2 & 11 & \\
\hline
\end{tabular}

*Fisher's exact test

***Pearson's chi-square test

${ }^{\text {a }}$ Significant relationship in single-variable analysis $(p<0.2)$ 
Table 2 Multiple logistic regression analysis results of factors for the development of deep vein thrombosis.

\begin{tabular}{|c|c|c|c|}
\hline Factor & Odds ratio & 95\% confidence interval & $p$ value \\
\hline Unilateral/bilateral & 4.751 & 1.23218 .320 & $0.024^{a}$ \\
\hline Hypertension & 2.591 & 0.7389 .098 & 0.137 \\
\hline Type of anesthesia & 4.210 & 1.07516 .482 & $0.039^{\mathrm{a}}$ \\
\hline Body mass index $\left(\mathrm{kg} / \mathrm{m}^{2}\right)$ & & & 0.142 \\
\hline$\geq 25$ & (Comparator) & & \\
\hline$\geq 25$ to $<30$ & 3.395 & 0.94412 .211 & 0.061 \\
\hline$\geq 30$ & 3.600 & 0.69118 .756 & 0.128 \\
\hline
\end{tabular}

${ }^{\text {a Significant factor in multiple logistic regression analysis }(p<0.05)}$

population was increasing, with one study reporting a DVT incidence of up to $35.7 \%$ without prophylaxis [6, 22]. A recent Japanese study also reported a $62.5 \%$ DVT rate after TKA without prophylaxis [24]. Furthermore, some studies even recommended medical prophylaxis to Korean patients having risk factors such as obesity [2]. Because of the increasing incidence of DVT and the legal problems when PE, which could be fatal, occurs, today an increasing number of surgeons in Korea now use medical prophylaxis for DVT after TKA $[6,29]$. We also acknowledge the risk of DVT and have used anticoagulant agents after TKA in our practice.
Several anticoagulant agents have been proposed for DVT prophylaxis after TKA. Today an increasing number of new agents have been invented and introduced to surgeons. Nonetheless, LMWH drugs such as enoxaparin and selective factor $\mathrm{Xa}$ inhibitors such as fondaparinux have been considered the standard therapy for prophylactic treatment for DVT $[6,8,9,25]$. We have chosen and used one of these standard materials, enoxaparin, for DVT prophylaxis after TKA. The efficacy and safety of LMWH is well documented in the literature [30]. However, there is a lack of literature reporting the DVT rate after TKA with chemoprophylaxis in the Asian

Table 3 Single-variable analysis results of factors for the development of deep vein thrombosis in unilateral cases

\begin{tabular}{|c|c|c|c|c|}
\hline Factor & & Positive & Negative & $p$ value \\
\hline Deep vein thrombosis & & 15 & 74 & \\
\hline \multirow[t]{2}{*}{ Gender } & Male & 4 & 7 & $0.085^{* a}$ \\
\hline & Female & 11 & 67 & \\
\hline \multirow[t]{3}{*}{ Age (years) } & $<65$ & 2 & 19 & $0.592^{* *}$ \\
\hline & $\geq 65$ to $<75$ & 12 & 33 & \\
\hline & $\geq 75$ & 1 & 22 & \\
\hline \multirow[t]{3}{*}{ Body mass index $\left(\mathrm{kg} / \mathrm{m}^{2}\right)$} & $<25$ & 3 & 33 & $0.021^{* * a}$ \\
\hline & $\geq 25$ to $<30$ & 8 & 35 & \\
\hline & $\geq 30$ & 4 & 6 & \\
\hline \multirow[t]{2}{*}{ Type of anesthesia } & Epidural & 3 & 35 & $0.084^{* a}$ \\
\hline & General & 12 & 39 & \\
\hline \multirow[t]{2}{*}{ Hypertension } & + & 12 & 44 & $0.156^{* a}$ \\
\hline & - & 3 & 30 & \\
\hline \multirow[t]{2}{*}{ Diabetes } & + & 3 & 19 & $0.754^{*}$ \\
\hline & - & 12 & 55 & \\
\hline \multirow[t]{2}{*}{ Hypercholesterolemia } & + & 3 & 7 & $0.363^{*}$ \\
\hline & - & 12 & 67 & \\
\hline \multirow[t]{4}{*}{ Prothrombin time/international normalized ratio } & $<0.95$ & 4 & 27 & $0.877^{* *}$ \\
\hline & $\geq 0.95$ to $<1.00$ & 5 & 20 & \\
\hline & $\geq 1.00$ to $<1.05$ & 4 & 16 & \\
\hline & $\geq 1.05$ & 2 & 11 & \\
\hline
\end{tabular}

*Fisher's exact test

***Pearson's chi-square test

${ }^{\text {a }}$ Significant relationship in single-variable analysis $(p<0.2)$ 
Table 4 Multiple logistic regression analysis results of factors for the development of deep vein thrombosis in unilateral cases

\begin{tabular}{|c|c|c|c|}
\hline Factor & Odds ratio & $95 \%$ confidence interval & $p$ value \\
\hline Gender & 3.646 & 0.76717 .329 & 0.104 \\
\hline Hypertension & 2.374 & 0.55210 .202 & 0.245 \\
\hline Type of anesthesia & 2.319 & 0.5449 .881 & 0.255 \\
\hline Body mass index $\left(\mathrm{kg} / \mathrm{m}^{2}\right)$ & & & 0.094 \\
\hline$\geq 25$ & (Comparator) & & \\
\hline$\geq 25$ to $<30$ & 2.333 & 0.52910 .284 & 0.263 \\
\hline$\geq 30$ & 8.057 & 1.22852 .863 & 0.030 \\
\hline
\end{tabular}

significant factor in multiple logistic regression analysis $(p<0.05)$

population. Chung et al. conducted a study with three different chemoprophylaxis regimens. The incidence rates of DVT were $7.3 \%, 15.5 \%$, and $35.5 \%$, respectively [6]. The DVT incidence rate of slightly more than $20 \%$ in our study is comparable with the results of Chung et al. and is lower than the results of previous studies without prophylaxis.

Risk factors for DVT after TKA have been studied and proposed by numerous investigators. Old age, female sex, obesity, and simultaneous bilateral cases of TKA are some of the well-known risk factors [2]. Rodgers et al. reported that epidural anesthesia reduced DVT incidence compared to general anesthesia [31]. Jiang et al. also reported that perioperative allogenic blood transfusion was significantly related to DVT following total joint arthroplasty [32]. However, there is a paucity of literature regarding DVT after TKA with the use of anticoagulation chemoprophylaxis in the Asian population. Because anticoagulant agents cannot prevent all cases of DVT after TKA, there should also be some risk factors for DVT even with prophylaxis. In this study, we found that general anesthesia and bilateral simultaneous cases were noticeable risk factors for DVT after TKA with chemoprophylaxis. Advanced age was not a significant factor, even in single-variable analyses. Although gender and BMI were notable factors in the single-variable analyses, they were not significant in the multiple logistic regression analyses.

There have been several studies comparing DVT incidence rates after TKA according to the anesthesia method. Most notably, $\mathrm{Hu}$ et al. reported in their metaanalysis study including 21 randomized controlled trials that epidural or spinal anesthesia significantly lowered the incidence of DVT when compared with general anesthesia. Their suggested reasons for this were altered coagulability, increased volume flow to the lower limbs, an improved ability to breathe without pain, and a reduction in surgical stress responses [33]. More recently, Pugely et al. reported no difference in DVT incidence rate between spinal anesthesia and general anesthesia. They used a large cohort database of more than 14,000 subjects: the American College of Surgeons National
Surgical Quality Improvement Program database between 2005 and 2010 [34]. Ishii et al. also reported that no anesthesia method was better than any other in relation to the development of symptomatic DVTs and PEs in a Japanese population [35]. Our study result backs the one stating that, even with chemoprophylaxis, general anesthesia increases the risk of DVT after TKA.

It has long been considered that simultaneous bilateral TKA cases increase DVT incidence compared to staged bilateral TKA cases. A meta-analysis study by $\mathrm{Fu}$ et al. compared various clinical outcomes between simultaneous bilateral TKA cases with staged bilateral TKA cases, and one of the findings was that simultaneous bilateral TKA cases were related to higher thromboembolism rates. They cited longer use of pneumatic tourniquets, intramedullary guides, and cement as factors for high DVT rates [36]. Recently, Liu et al. reported in their meta-analysis study including 18 comparative studies that simultaneous bilateral TKA cases showed increased DVT incidence rates compared with staged TKAs [37]. Our study compared simultaneous bilateral TKA cases with only first leg staged bilateral cases or cases that planned to have just unilateral cases. We also found that simultaneous bilateral TKA increases the risk of DVT.

For the diagnosis of DVT, several modalities are available. Because the clinical diagnosis of DVT is somewhat difficult because of the vague symptoms, radiologic and laboratory tests are usually required. The serum Ddimer test has been considered as a useful screening test; however, because of its poor specificity, it has a limited value as a definite diagnostic method $[2,38]$. Compression ultrasonography was reported to be safe and effective; however, there are concerns of accuracy issues [39]. Today CT angiography is widely considered a more sensitive tool in detecting DVT [40, 41]. We used CT angiography to secure an accurate diagnosis.

As for the timing of the DVT evaluation, the protocol in place for this study was the seventh postoperative day. There have been some variances among studies; however, most performed their DVT evaluation about seven days after the TKA. Kim et al. reported their results 
based on the sixth postoperative day DVT evaluations [2], and Park et al. reported the seventh postoperative day results [5]. Chung et al. stated that they performed the evaluation before the 10th postoperative day [6].

\section{Limitations}

There are several limitations to this study. First, the risk factors for DVT that we used were selected rather arbitrarily. However, we referred to the literature for the selection of factors, and some of them were common choices for general clinical factor analysis studies. Second, a sample size of 103 was small for a factor analysis study. In retrospective observational studies like this one, a bigger sample size is always desirable. We hope to someday run a similar study with a much larger sample size. Third, because we did not perform lower extremity CT angiography before the operation, we could not differentiate whether the detected DVTs were old lesions or newly developed lesions after TKA. However, Chang et al. reported that preoperative DVT was rare and of limited clinical relevance [40]. Fourth, we did not investigate PE occurrence. If we had these data, it would have been a more complete study. However, because of the rareness of PE after TKA in Koreans [1, 14, 19], we suspected that it would be meaningless to investigate the $\mathrm{PE}$ occurrence with this sample size. Lastly, we did not collect data on patient symptoms related to DVT from their medical records. It would have been a much better and more complete study if we had presented data and analyses of DVT symptoms. Unfortunately, because this was a retrospective study, we could not collect reliable and constant data on symptoms in patients with DVT from the medical records.

\section{Conclusions}

Patients who undergo simultaneous bilateral TKAs and patients who receive general anesthesia need more attention regarding DVT, even with DVT chemoprophylaxis using LMWH after TKA.

\section{Abbreviations \\ DVT: deep vein thrombosis; TKA: total knee arthroplasty; LMWH: low- molecular-weight-heparin; ROM: range of motion; CT: computed tomography; BMI: body mass index; PT INR: prothrombin time/international normalized ratio}

\section{Acknowledgements}

None.

\section{Authors' contributions}

JKL conceptualized the study; treated the subjects; gathered, analyzed, and interpreted the subject data; and was a major contributor in writing, reviewing, and editing the manuscript. KBL conceptualized the study, treated the subjects, and supervised the study. JIK conceptualized the study, edited the manuscript, and supervised the study. GTP gathered the subject data and contributed in writing the manuscript. YCC contributed in writing and editing the manuscript. The authors read and approved the final manuscript.
Funding

None.

Availability of data and materials

The complete datasets used in this study are available from the corresponding author on adequate request.

\section{Declarations}

Ethics approval and consent to participate

IRB No. 2017-I140. Title: Risk Factors of Deep Vein Thrombosis after Total Knee Arthroplasty.

Consent for publication

This was undertaken by the institutional consent form.

\section{Competing interests}

The authors declare that they have no competing interests.

\section{Author details}

'Department of Orthopaedic Surgery, Konkuk University Medical Center, 120-1, Neungdong-ro, Gwangjin-gu, Seoul 05030, South Korea. ${ }^{2}$ Research Institute of Medical Science, Konkuk University School of Medicine, 120-1, Neungdong-ro, Gwangjin-gu, Seoul 05030, South Korea. ${ }^{3}$ Department of Orthopaedic Surgery, Cheongju St. Mary's Hospital, 173-19 Jusung-ro, Cheongwon-gu, Cheongju-si, Chungcheongbuk-do 28323, South Korea. ${ }^{4}$ Department of Orthopaedic Surgery, Hallym University Kangnam Sacred Heart Hospital, 1, Singil-ro, Yeongdeungpo-gu, Seoul 07741, South Korea. ${ }^{5}$ Department of Orthopaedic Surgery, Hallym University Sacred Heart Hospital, 22 Gwanpyeong-ro, 170beon-gil, Dongan-gu, Anyang-si, Gyeonggi-do 14068, South Korea.

Received: 23 March 2021 Accepted: 12 July 2021

Published online: 07 September 2021

\section{References}

1. Piovella F, Wang CJ, Lu H, Lee K, Lee LH, Lee WC et al (2005) Deep-vein thrombosis rates after major orthopedic surgery in Asia. An epidemiological study based on postoperative screening with centrally adjudicated bilateral venography. J Thromb Haemost 3(12):2664-2670. https://doi.org/10.1111/j.1 538-7836.2005.01621.x

2. Kim Kl, Cho KY, Jin W, Khurana SS, Bae DK (2011) Recent Korean perspective of deep vein thrombosis after total knee arthroplasty. J Arthroplast 26(7): 1112-1116. https://doi.org/10.1016/j.arth.2011.02.021

3. Harrison-Brown M, Scholes C, Douglas SL, Farah SB, Kerr D, Kohan L (2020) Multimodal thromboprophylaxis in low-risk patients undergoing lower limb arthroplasty: a retrospective observational cohort analysis of 1400 patients with ultrasound screening. J Orthop Surg (Hong Kong) 28(2): 2309499020926790

4. Lee WS, Kim Kl, Lee HJ, Kyung HS, Seo SS (2013) The incidence of pulmonary embolism and deep vein thrombosis after knee arthroplasty in Asians remains low: a meta-analysis. Clin Orthop Relat Res 471(5):1523-1532. https://doi.org/10.1007/s11999-012-2758-9

5. Park SH, Ahn JH, Park YB, Lee SG, Yim SJ (2016) Incidences of deep vein thrombosis and pulmonary embolism after total knee arthroplasty using a mechanical compression device with and without low-molecular-weight heparin. Knee Surg Relat Res 28(3):213-218. https://doi.org/10.5792/ksrr.201 6.28.3.213

6. Chung KS, Shin TY, Park SH, Kim H, Choi CH (2018) Rivaroxaban and acetylsalicylic acid for prevention of venous thromboembolism following total knee arthroplasty in Korean patients. Knee Surg Relat Res. 30(3):247254. https://doi.org/10.5792/ksrr.17.092

7. Masuda EM, Kistner RL, Musikasinthorn C, Liquido F, Geling O, He Q (2012) The controversy of managing calf vein thrombosis. J Vasc Surg 55(2):550561. https://doi.org/10.1016/j.jvs.2011.05.092

8. Sasaki H, Ishida K, Shibanuma N, Tei K, Tateishi H, Toda A, Yamashiro Y, Matsumoto T, Kuroda R, Kurosaka M (2014) Retrospective comparison of three thromboprophylaxis agents, edoxaban, fondaparinux, and enoxaparin, for preventing venous thromboembolism in total knee arthroplasty. Int Orthop 38(3):525-529. https://doi.org/10.1007/s00264-013-2132-x 
9. Beyer-Westendorf J, Lutzner J, Donath L, Radke OC, Kuhlisch E, Hartmann A et al (2012) Efficacy and safety of rivaroxaban or fondaparinux thromboprophylaxis in major orthopedic surgery: findings from the ORTHOTEP registry. J Thromb Haemost 10(10):2045-2052. https://doi.org/10.1111/ j.1538-7836.2012.04877.x

10. Fuji T, Wang CJ, Fujita S, Kawai Y, Nakamura M, Kimura T, Ibusuki K, Ushida H, Abe K, Tachibana S (2014) Safety and efficacy of edoxaban, an oral factor Xa inhibitor, versus enoxaparin for thromboprophylaxis after total knee arthroplasty: the STARS E-3 trial. Thromb Res 134(6):1198-1204. https://doi. org/10.1016/j.thromres.2014.09.011

11. Kim YH, Anil V, Gaurav A, Park JW, Kim JS (2019) Mechanical thromboprophylaxis would suffice after total knee arthroplasties in Asian patients? Arch Orthop Trauma Surg 139(2):167-171. https://doi.org/10.1007/ s00402-018-3045-3

12. Cho KY, Kim Kl, Khurana S, Bae DK, Jin W (2013) Is routine chemoprophylaxis necessary for prevention of venous thromboembolism following knee arthroplasty in a low incidence population? Arch Orthop Trauma Surg 133(4):551-559. https://doi.org/10.1007/s00402-013-1691-z

13. Clarke MT, Green JS, Harper WM, Gregg PJ (1997) Screening for deep-venous thrombosis after hip and knee replacement without prophylaxis. J Bone Joint Surg Br 79(5):787-791. https://doi.org/10.1302/0301-620X.79B5.0790787

14. Kim YH (1990) The incidence of deep vein thrombosis after cementless and cemented knee replacement. J Bone Joint Surg Br. 72(5):779-783

15. Geerts WH, Pineo GF, Heit JA, Bergqvist D, Lassen MR, Colwell CW, Ray JG (2004) Prevention of venous thromboembolism: the Seventh ACCP Conference on Antithrombotic and Thrombolytic Therapy. Chest. 126(3 Suppl):338S-400S. https://doi.org/10.1378/chest.126.3_suppl.338S

16. McKenna R, Bachmann F, Kaushal SP, Galante JO (1976) Thromboembolic disease in patients undergoing total knee replacement. J Bone Joint Surg Am 58(7):928-932. https://doi.org/10.2106/00004623-197658070-00006

17. Lotke PA, Steinberg ME, Ecker ML (1994) Significance of deep venous thrombosis in the lower extremity after total joint arthroplasty. Clin Orthop Relat Res 299:25-30

18. White RH, Keenan CR (2009) Effects of race and ethnicity on the incidence of venous thromboembolism. Thromb Res 123(Suppl 4):S11-S17. https://doi. org/10.1016/S0049-3848(09)70136-7

19. Kim YH, Yoo JH, Kim JS (2007) Factors leading to decreased rates of deep vein thrombosis and pulmonary embolism after total knee arthroplasty. J Arthroplast 22(7):974-980. https://doi.org/10.1016/j.arth.2007.03.033

20. Kim YH, Oh SH, Kim JS (2003) Incidence and natural history of deep-vein thrombosis after total hip arthroplasty. A prospective and randomised clinical study. J Bone Joint Surg Br. 85(5):661-665

21. Zhang S, Htet KS, Tan XY, Wang X, Wang W, Chua W (2020) Short-duration chemoprophylaxis might reduce incidence of deep vein thrombosis in Asian patients undergoing total knee arthroplasty. Knee Surg Relat Res. 32(1):58. https://doi.org/10.1186/s43019-020-00077-w

22. Park KH, Cheon SH, Lee JH, Kyung HS (2012) Incidence of venous thromboembolism using 64 channel multidetector row computed tomography-indirect venography and anti-coagulation therapy after total knee arthroplasty in Korea. Knee Surg Relat Res. 24(1):19-24. https://doi. org/10.5792/ksrr.2012.24.1.19

23. Leizorovicz A, Committee SVSS (2007) Epidemiology of post-operative venous thromboembolism in Asian patients. Results of the SMART venography study. Haematologica. 92(9):1194-1200. https://doi.org/10.3324/ haematol.10819

24. Tateiwa T, Ishida T, Masaoka T, Shishido T, Takahashi Y, Onozuka A et al (2019) Clinical course of asymptomatic deep vein thrombosis after total knee arthroplasty in Japanese patients. J Orthop Surg (Hong Kong) 27(2): 2309499019848095

25. Falck-Ytter $Y$, Francis CW, Johanson NA, Curley C, Dahl OE, Schulman S, Ortel TL, Pauker SG, Colwell CW Jr (2012) Prevention of VTE in orthopedic surgery patients: Antithrombotic Therapy and Prevention of Thrombosis, 9th ed: American College of Chest Physicians Evidence-Based Clinical Practice Guidelines. Chest. 141(2 Suppl):e278S-e325S. https://doi.org/10.1378/ chest.11-2404

26. Melese KG, Weji BG, Berheto TM, Bekru ET (2020) Utilization of partograph during labour: a case of Wolaita Zone, Southern Ethiopia. Heliyon 6(12): e05633. https://doi.org/10.1016/j.heliyon.2020.e05633

27. Osiewicz M, Lobbezoo F, Ciapala B, Pytko-Polonczyk J, Manfredini D (2020) Pain predictors in a population of temporomandibular disorders patients. J Clin Med 9(2):452
28. Takigawa T, Tanaka M, Sugimoto Y, Tetsunaga T, Nishida K, Ozaki T (2017) Discrimination between malignant and benign vertebral fractures using magnetic resonance imaging. Asian Spine J 11(3):478-483. https://doi.org/1 0.4184/asj.2017.11.3.478

29. Barrack RL (2012) Current guidelines for total joint VTE prophylaxis: dawn of a new day. J Bone Joint Surg Br 94(11 Suppl A):3-7

30. Sun G, Wu J, Wang Q, Liang Q, Jia J, Cheng K, Sun G, Wang Z (2019) Factor Xa inhibitors and direct thrombin inhibitors versus low-molecular-weight heparin for thromboprophylaxis after total hip or total knee arthroplasty: a systematic review and meta-analysis. J Arthroplast 34(4):789-800 e6. https:// doi.org/10.1016/j.arth.2018.11.029

31. Rodgers A, Walker N, Schug S, McKee A, Kehlet H, van Zundert A, Sage D, Futter M, Saville G, Clark T, MacMahon S (2000) Reduction of postoperative mortality and morbidity with epidural or spinal anaesthesia: results from overview of randomised trials. BMJ. 321(7275):1493. https://doi.org/10.1136/ bmj.321.7275.1493

32. Jiang T, Song K, Yao Y, Pan P, Jiang Q (2019) Perioperative allogenic blood transfusion increases the incidence of postoperative deep vein thrombosis in total knee and hip arthroplasty. J Orthop Surg Res 14(1):235. https://doi. org/10.1186/s13018-019-1270-2

33. Hu S, Zhang ZY, Hua YQ, Li J, Cai ZD (2009) A comparison of regional and general anaesthesia for total replacement of the hip or knee: a metaanalysis. J Bone Joint Surg Br. 91(7):935-942

34. Pugely AJ, Martin CT, Gao Y, Mendoza-Lattes S, Callaghan JJ (2013) Differences in short-term complications between spinal and general anesthesia for primary total knee arthroplasty. J Bone Joint Surg Am 95(3): 193-199. https://doi.org/10.2106/JBJS.K.01682

35. Ishii Y, Noguchi H, Sato J, Takayama S, Okada Y, Toyabe SI (2018) Impact of anesthesia modality and mechanical venous thromboembolism prophylaxis on the incidence of symptomatic deep venous thrombosis after TKA. J Clin Orthop Trauma 9(2):142-145. https://doi.org/10.1016/j.jcot.2016.10.013

36. Fu D, Li G, Chen K, Zeng H, Zhang X, Cai Z (2013) Comparison of clinical outcome between simultaneous-bilateral and staged-bilateral total knee arthroplasty: a systematic review of retrospective studies. J Arthroplast 28(7): 1141-1147. https://doi.org/10.1016/j.arth.2012.09.023

37. Liu L, Liu H, Zhang H, Song J, Zhang L (2019) Bilateral total knee arthroplasty: simultaneous or staged? A systematic review and metaanalysis. Medicine (Baltimore) 98(22):e15931. https://doi.org/10.1097/MD 0000000000015931

38. Yoo MC, Cho YJ, Ghanem E, Ramteke A, Kim KI (2009) Deep vein thrombosis after total hip arthroplasty in Korean patients and D-dimer as a screening tool. Arch Orthop Trauma Surg 129(7):887-894. https://doi.org/1 0.1007/s00402-008-0751-2

39. Tan M, van Rooden CJ, Westerbeek RE, Huisman MV (2009) Diagnostic management of clinically suspected acute deep vein thrombosis. $\mathrm{Br} J$ Haematol 146(4):347-360. https://doi.org/10.1111/j.1365-2141.2009.07732x

40. Chang MJ, Song MK, Kyung MG, Shin JH, Chang CB, Kang SB (2018) Incidence of deep vein thrombosis before and after total knee arthroplasty without pharmacologic prophylaxis: a 128-row multidetector $C T$ indirect venography study. BMC Musculoskelet Disord 19(1):274. https://doi.org/1 0.1186/s12891-018-2166-8

41. Shin WC, Woo SH, Lee SJ, Lee JS, Kim C, Suh KT (2016) Preoperative prevalence of and risk factors for venous thromboembolism in patients with a hip fracture: an indirect multidetector $C T$ venography study. J Bone Joint Surg Am 98(24):2089-2095. https://doi.org/10.2106/JBJS.15.01329

\section{Publisher's Note}

Springer Nature remains neutral with regard to jurisdictional claims in published maps and institutional affiliations. 